\title{
Tailoring Surface Chemistry of Sugar-Derived Ordered Mesoporous Carbons towards Efficient Removal of Diclofenac from Aquatic Environments
}

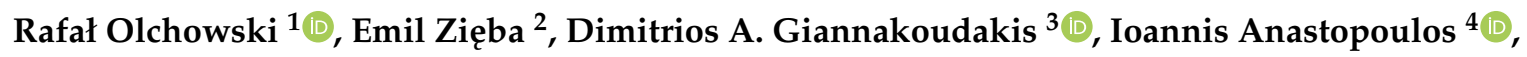 \\ Ryszard Dobrowolski ${ }^{1}$ (D) and Mariusz Barczak ${ }^{5, *(1)}$ \\ 1 Department of Analytical Chemistry, Institute of Chemical Sciences, Faculty of Chemistry, Maria \\ Curie-Skłodowska University in Lublin, 20-031 Lublin, Poland; \\ rafal.olchowski@poczta.umcs.lublin.pl (R.O.); rdobrow@poczta.umcs.lublin.pl (R.D.) \\ 2 Confocal and Electron Microscopy Laboratory, Center for Interdisciplinary Research, John Paul II Catholic \\ University of Lublin, Konstantynów Sq. 1J, 20-708 Lublin, Poland; dzemski@gmail.com \\ 3 Department of Chemistry, Aristotle University of Thessaloniki, 54-124 Thessaloniki, Greece; \\ dagchem@gmail.com \\ 4 Department of Chemistry, University of Cyprus, P.O. Box 20537, Nicosia CY-1678, Cyprus; \\ anastopoulos_ioannis@windowslive.com \\ 5 Department of Theoretical Chemistry, Institute of Chemical Sciences, Faculty of Chemistry, Maria \\ Curie-Skłodowska University in Lublin, 20-031 Lublin, Poland \\ * Correspondence: mbarczak@umcs.pl; Tel.: +48-81-537-7992
}

Received: 29 February 2020; Accepted: 28 March 2020; Published: 1 April 2020

\begin{abstract}
Ordered mesoporous carbon (CMK-3), obtained from an abundant natural source, sugar, was thermochemically modified with dicyandiamide and thiourea as well as by classical oxidization with hydrogen peroxide to introduce specific surface groups. Thermochemical modifications resulted in carbon with almost unchanged porosity and altered surface chemistry while porosity of $\mathrm{H}_{2} \mathrm{O}_{2}$-treated carbon was seriously deteriorated. The obtained carbons were tested as sorbents of diclofenac, considered as one of the emerging water contaminants. Changes in porosity and surface chemistry of modified carbons resulted in significant differences with regard to the uptake of diclofenac. Dicyandiamide-modified carbon showed highest uptake of drugs, reaching $241 \mathrm{mg}$ $\mathrm{g}^{-1}$ that is attributed to its developed microporosity as well as surface chemistry composed of basic groups facilitating electrostatic interactions with diclofenac anions. Desorption study showed that diclofenac is strongly bonded, albeit with a different degree depending on the modification of the CMK-carbon. The obtained results were compared with up-to-date literature regarding sorption of diclofenac by carbon-based sorbents.
\end{abstract}

Keywords: mesoporous carbon; bioresources; water remediation; adsorption; pharmaceuticals

\section{Introduction}

Ordered mesoporous carbons (OMCs) have been widely studied due to numerous advantages, including large surface areas and well defined in shape and size mesopores, as well as the possibility of further structural or chemical surface modification. OMCs can be considered as prosperous green-oriented materials for environmental remediation applications, since they can be derived from an abundant natural source, sugar/sucrose. The first fabrication protocols were based on the hard-template method (also called nanocasting) which involves replication of the presynthesized mesoporous silica scaffolds by filling its pores with organic precursor followed by carbonization and removal of sacrificial template [1-3]. Simultaneously, another protocol was developed where micelles of amphiphilic 
surfactant (usually triblock copolymer Pluronic P123) are acting as the template for the organic precursor [4]. Pyrolytic removal of the sacrificial template results in a carbon structure with mesopores. This self-assembly route is known as soft-templating. Theoretically, a plethora of organic precursors can be used, however, most frequently, phenol, resorcinol, formaldehyde, or carbohydrates are preferred. Apart from the two principal fabrication strategies mentioned above, there are some alternative ways to obtain mesoporous carbons, including mechanochemically induced self-assembly [5], sol-gel method [6], or other approaches [7]. Some of them were also developed to produce ordered porous carbon films [8].

Perspectives for potential applications of mesoporous templated carbons are broad and include, among others, removal of hazardous pollutants [9,10], catalytic processes [11,12], energy storage [13-15], and controlled drug delivery systems [16,17], as well as bioadsorption and biocatalysis [18,19]. The hydrophobic nature of OMCs makes them often poorly suited for removal of different species from aqueous systems due to the limited wetting of the sorbent surface resulting in restricted penetration of the pores by the molecules intended to be adsorbed [20]. In such cases, surface modification with appropriate surface functional groups is a strategy to overcome those difficulties [21]. A comparative study on adsorption of several nonsteroidal anti-inflammatory drugs by activated carbon and soft-templated OMCs revealed that the pristine and $\mathrm{CO}_{2}$ oxidized mesoporous carbons have significantly better adsorption performance than classic microporous activated carbon. The adsorption mechanism was explained by $\pi$ electron interactions between the widely used anti-inflammatory drug ibuprofen/naproxen and carbon surface because of the possibility of forming Lewis acid-base complexes or hydrogen bonds [22]. Granular OMC was used for removal of several antibiotics, and the obtained carbons again proved to be better sorbents than classic activated carbon. The proposed adsorption mechanism was explained by complexation reactions and dispersive forces between the $\pi-\pi^{*}$ electrons in the graphene layers of the carbon and tetracycline aromatic rings [23]. Another interesting study on adsorption of antibiotics showed that OMC materials can provide fast adsorption kinetics and relevant adsorption uptakes. The strong adsorption of tetracycline was explained by the interaction of its $\mathrm{O}$ and $\mathrm{N}$ groups (e.g., phenol, amine) with the graphene surface [24].

To date, several modification protocols have been applied in order to manipulate the surface chemistry heterogeneity and structural features of ordered mesoporous carbons. Most of them are based on chemical oxidation achieved by using different agents, like $\left(\mathrm{NH}_{4}\right)_{2} \mathrm{~S}_{2} \mathrm{O}_{8}, \mathrm{H}_{2} \mathrm{O}_{2}, \mathrm{H}_{3} \mathrm{PO}_{4}$, or $\mathrm{HNO}_{3}$, which leads to the formation of oxygen functional groups (i.e., carbonyl, carboxyl, hydroxyl, phenol, and lactone ones) during the oxidation process [10,25]. Although $\mathrm{HNO}_{3}$ oxidation can result in the formation of N-containing functional groups, they are often unstable [26]. Another approach relies on the use of the heteroatoms-rich metal-free precursor for synthesis of OMC resulting in fabrication of mesoporous carbons with elevated content of heteroatom functionalities [26,27]. The main disadvantages of the latter method are: (1) Higher cost of the final material due to the use of precursors, which are usually more expensive than classical precursors, and (2) emissions of hazardous compounds during pyrolysis, like $\mathrm{HCN}$ or $\mathrm{NH}_{3}$ [26].

It is often reported that the introduction of surface functional groups can significantly enhance the sorption capability against targeted hazardous compounds. Nevertheless, the modification strategies beyond classical oxidation treatments are rarely reported, despite the fact that heteroatoms, like sulfur, nitrogen, or oxygen, can generate surface polarity [26]. Surface chemistry of the OMC materials is similar to that of classical activated carbons, so the methods followed for activation of classical carbon-based sorbents (e.g., microporous activated carbons) could be also utilized to modify OMCs. Among them, thermal treatment of the carbon materials previously impregnated with substances bearing desired heteroatoms is among the most efficient, however, not widely applied strategies. Such thermochemical treatment can lead to a remarkable doping of the carbon structure while keeping its mechanical and structural properties unchanged.

In this paper, we report the synthesis of ordered mesoporous carbon CMK-3 (Carbon Mesostructured by KAIST—Korea Advanced Institute of Science and Technology—Number 3) and its subsequent modification 
using two paths: (1) Classical low-temperature wet oxidation using $\mathrm{H}_{2} \mathrm{O}_{2}$ and (2) carbonization at $800{ }^{\circ} \mathrm{C}$ of dicyandiamide- and thiourea-impregnated CMK-3 carbons. The effects of the modification treatment on the resulting surface chemistry and porosity of the adsorption performances of the obtained carbons were also investigated. Diclofenac was chosen as model adsorbate since it is considered as one of the most hazardous pollutants representing a new class of emerging contaminants (pharmaceuticals). Nonsteroidal anti-inflammatory drugs like diclofenac are of huge concern due to their widespread use and intensive discards mainly from households and hospitals [28]. To the best of our knowledge, modified OMCs have not been tested yet as potential sorbents of diclofenac so this work is the first attempt to study in detail the adsorption of this drug. Another novelty of this study is that not-yet-explored postsynthesis modification of OMCs by dicyandiamide and thiourea was thoroughly investigated to verify if the adsorptive capabilities of modified carbons can be altered upon the surface chemistry and structural alterations.

\section{Materials and Methods}

\subsection{Reagents}

The following reagents were used as received: Pluronic P123 (P123, Sigma-Aldrich, Saint Louis, MO, USA), tetraethoxysilane (TEOS, 99\%, ABCR GmbH, Karlsruhe, Germany), HCl (36\%, POCH, Polish Chemical Reagents, Gliwice, Poland), ethanol (EtOH, 99.8\%, POCH, Polish Chemical Reagents, Gliwice, Poland), $\mathrm{NaOH}$ (POCH, Polish Chemical Reagents, Gliwice, Poland), $\mathrm{H}_{2} \mathrm{SO}_{4}(96 \%, \mathrm{Merck}$ KGaA, Darmstadt, Germany), sucrose (food sugar, Pfeifer \& Langen GmbH \& Co. KG, Cologne, Germany), $\mathrm{H}_{2} \mathrm{O}_{2}$ (30\%, Sigma-Aldrich, Saint Louis, MO, USA), dicyandiamide (99\%, Sigma-Aldrich, Saint Louis, MO, USA), thiourea (>99\%, Sigma-Aldrich, Saint Louis, MO, USA), diclofenac sodium salt (DICL, >98\%, Sigma-Aldrich, Saint Louis, MO, USA). The above-mentioned chemicals were used as received, without further purification. Deionized water (DW, resistivity $<17.5 \mathrm{mV} \mathrm{cm}$ ) was obtained from a Millipore system (Merck Millipore, Burlington, MA, USA).

\subsection{Synthesis of the CMK-3 Carbon and its Further Modification}

\subsubsection{Synthesis of SBA-15 Template}

Synthesis of SBA-15 (Santa Barbara Amorphous Number 15) template was conducted by the procedure described below. Five grams of the symmetric triblock copolymer P123 was dissolved in $180 \mathrm{~mL} \mathrm{HCl}$ solution $\left(2 \mathrm{M}\left(\mathrm{mol} \mathrm{L}^{-1}\right)\right)$ under vigorous stirring $(500 \mathrm{rpm})$ at $40{ }^{\circ} \mathrm{C}$. After complete dissolution of P123, $50 \mathrm{mmol}$ of TEOS was added dropwise during $150 \mathrm{~s}$. The mixture was stirred for $24 \mathrm{~h}$, transferred into the oven and aged for $48 \mathrm{~h}$ at $100{ }^{\circ} \mathrm{C}\left(2{ }^{\circ} \mathrm{C} \mathrm{min}-1\right)$ under static conditions. After the hydrothermal treatment, the solid material was recovered by filtration and subjected to a triple extraction by $255 \mathrm{~mL}$ of $0.2 \mathrm{M} \mathrm{HCl}$ in ethanol at $78{ }^{\circ} \mathrm{C}$ for $6 \mathrm{~h}$. During the extraction process the suspension was stirred manually every $30 \mathrm{~min}$. Finally, the received material was thoroughly washed by ethanol and water (until reaching the neutral $\mathrm{pH}$ of the filtrate), followed by drying at $100{ }^{\circ} \mathrm{C}$ for $24 \mathrm{~h}$. Physicochemical characterization of SBA-15 template is presented in Figure S1.

\subsubsection{Synthesis of Pristine CMK-3}

First, $7.5 \mathrm{~g}$ of sucrose was dissolved in $30 \mathrm{~mL}$ of water containing $600 \mu \mathrm{L}$ of $98 \% \mathrm{H}_{2} \mathrm{SO}_{4}$ under stirring at $300 \mathrm{rpm}$, followed by the addition of $6 \mathrm{~g} \mathrm{SBA}-15$. The mixture was heated at $100{ }^{\circ} \mathrm{C}$ for $6 \mathrm{~h}$ in an oven and then the temperature was raised to $160{ }^{\circ} \mathrm{C}\left(2{ }^{\circ} \mathrm{C} \min ^{-1}\right)$ for another $6 \mathrm{~h}$. The impregnation with sucrose was repeated by using a solution of sucrose in water $(6 \mathrm{~g}$ of sucrose and $37.8 \mathrm{~mL}$ of water) and $385.8 \mu \mathrm{L}$ of $98 \% \mathrm{H}_{2} \mathrm{SO}_{4}$. Once again, the mixture was kept at $100{ }^{\circ} \mathrm{C}$ for $6 \mathrm{~h}$ and at $160{ }^{\circ} \mathrm{C}$ $\left(2{ }^{\circ} \mathrm{C} \mathrm{min}-1\right)$ for another $6 \mathrm{~h}$. The obtained Si/C composite was carbonized at $900{ }^{\circ} \mathrm{C}$ for $3 \mathrm{~h}$ under nitrogen flow $\left(1 \mathrm{~L} \mathrm{~min}^{-1}\right)$. Heating rate was $10^{\circ} \mathrm{C} \mathrm{min}{ }^{-1}$. SBA-15 template was removed by shaking the sample with $1 \mathrm{M}$ ethanol/water solution (volume ratio 1:1) of $\mathrm{NaOH}$ for 5 hours at $100{ }^{\circ} \mathrm{C}$. After that, the sample was thoroughly washed with $1 \mathrm{M} \mathrm{HCl}$ and water until $\mathrm{Na}$ concentration in filtrate was 
$<1 \mathrm{mg} \mathrm{L}^{-1}$ (measured by F AAS (Flame Atomic Absorption Spectrometry) technique), followed by drying at $120^{\circ} \mathrm{C}$ for $24 \mathrm{~h}$. This unmodified sample was denoted as P-CMK.

\subsubsection{Wet Oxidation of CMK-3}

First, $0.2 \mathrm{~g}$ of CMK-3 was placed into a beaker filled with $10 \% \mathrm{H}_{2} \mathrm{O}_{2}(50 \mathrm{~mL})$ and the mixture was heated for $4 \mathrm{~h}$ at $90^{\circ} \mathrm{C}$ in a water bath under stirring at $300 \mathrm{rpm}$. Afterwards, the mixture was filtered and thoroughly washed with deionized water. The obtained sample was dried at $120^{\circ} \mathrm{C}$ for $24 \mathrm{~h}$. This oxidized sample is abbreviated as H-CMK in the text.

\subsubsection{Dicyandiamide- and Thiourea-Modification of CMK-3}

First, $1 \mathrm{~g}$ of CMK-3 was added to a $120 \mathrm{~mL}$ aqueous solution containing $4.2 \mathrm{~g}$ of dicyandiamide or $16.6 \mathrm{~g}$ of thiourea. The carbon slurries were stirred at room temperature for $24 \mathrm{~h}$. Next, the impregnated solids were recovered by filtration and dried at $80^{\circ} \mathrm{C}$ for $24 \mathrm{~h}$. Finally, they were carbonized at $800^{\circ} \mathrm{C}$ (rate: $10^{\circ} \mathrm{C} \mathrm{min}^{-1}$ ) for $40 \mathrm{~min}$ under $\mathrm{N}_{2}$ flow. Sample modified by dicyandiamide is denoted as D-CMK and by thiourea as T-CMK.

\subsection{Instrumental Characterization}

The nitrogen isotherms were measured at $-196{ }^{\circ} \mathrm{C}$ using a Quantachrome 1200e analyzer (Quantachrome Instruments, Boynton Beach, FL, USA) after degassing the samples at $120^{\circ} \mathrm{C}$ in vacuum for $12 \mathrm{~h}$. The micropore $\left(\mathrm{V}_{\text {mic }}\right)$ and mesopore $\left(\mathrm{V}_{\text {mes }}\right)$ total pore $\left(\mathrm{V}_{\text {tot }}\right)$ volumes and pore size distributions (PSD) were estimated using the desorption branch of the isotherm by quenched solid density functional theory method (QSDFT) with a slit-shaped pore model applied [29], using ASiQwin 3.0 software (Quantachrome Instruments, Boynton Beach, FL, USA). The zeta potential was evaluated using a Zetasizer Nano ZS (Malvern Instruments, Malvern, UK). Suspensions were prepared by dispersing $2.0 \mathrm{mg}$ of ball-milled samples in $2 \mathrm{~mL}$ of $1 \mathrm{mM}\left(\mathrm{mmol} \mathrm{L}^{-1}\right) \mathrm{KCl}$. The $\mathrm{pH}$ of an aqueous solution of drug or $1 \mathrm{mM} \mathrm{KCl}$ was adjusted by addition of the proper amount of $1 \mathrm{M} \mathrm{HCL}$ or $1 \mathrm{M} \mathrm{NaOH}$ solutions. The $\mathrm{pH}$ measurements were accomplished using $\mathrm{pH}$ meter CP-401 (Elmetron, Zabrze, Poland) equipped with a glass electrode after proper calibration. X-ray photoelectron spectroscopy (XPS) measurements were performed on a Multi-Chamber Analytical System (Prevac, Rogów, Poland) equipped with monochromatic $\mathrm{K}_{\alpha}-\mathrm{Al}$ radiation (1486.6 eV (electron volt)) (Gammadata Scienta, Uppsala, Sweden) and an X-ray power of $450 \mathrm{~W}$. Binding energies were referenced to the carbon C1s peak at $285 \mathrm{eV}$. Empyrean (PANalytical, Malvern, UK) diffractometer $\left(\mathrm{CuK}_{\alpha}\right.$ radiation) working with $0.02^{\circ}$ size step and $10 \mathrm{~s}$ time step was used to record powder $\mathrm{X}$-ray diffraction (XRD) patterns. The inspection of the surface of the CMK-3 was done by using the scanning electron microscope Carl Zeiss Ultra Plus (Carl Zeiss, Jena, Germany) equipped with an energy dispersive X-ray detector BrukerAXS (Bruker, Karlsruhe, Germany). The microscope was also equipped with secondary electron (SE) and backscattered electron (BSE) detectors. All experiments were carried out under required conditions (20-kV acceleration voltage and 5-nA probe current). Raman spectra were measured on an inVia Reflex (Renishaw, Wotton-under-Edge, UK) dispersive Raman microscope with an ion-argon laser $(514 \mathrm{~nm}, 20 \mathrm{~mW})$. Sodium concentration in water solutions was measured by flame atomic absorption spectrometer (F AAS) (SpectrAA 880, Varian, Australia) equipped with an air-acetylene burner. Spectral bandwidth of $0.5 \mathrm{~nm}$, acetylene flow rate of $3.0 \mathrm{~L}$ $\mathrm{min}^{-1}$, and air flow rate of $13.5 \mathrm{~L} \mathrm{~min}^{-1}$ were conventional working parameters. F AAS measurements were performed using Na hollow cathode lamp (Varian, Australia) at wavelength $589.0 \mathrm{~nm}, 0.5-\mathrm{nm}$ slit, and 5-mA lamp current.

\subsection{Sorption of DICL}

In each experiment, $10 \mathrm{mg}$ of adsorbent was added to $20 \mathrm{~mL}$ of an aqueous diclofenac (DICL) solution and shaken using MaxQ ${ }^{\mathrm{TM}}$ Mini 4450 orbital shaker (Waltham, MA, USA) (150 rpm) for $48 \mathrm{~h}$ (only in the case of kinetics measurements, contact times varied to collect kinetic curves, namely 0.5, 1 , 
$2,5,12,24,48 \mathrm{~h})$. The initial DICL concentrations used to collect isotherm data were: $25,50,75,100$, $150,250,400 \mathrm{mg} \mathrm{L}^{-1}$. The adsorbed amounts were calculated from the mass balance according to the formula, $a=\left(c_{i}-c_{j}\right) \cdot V \cdot m^{-1}$, where $a$ is adsorbed amount $\left(\mathrm{mg} \mathrm{g}^{-1}\right), c_{i}$ is the initial DICL concentration $\left(\mathrm{mg} \mathrm{L}^{-1}\right), \mathrm{c}_{\mathrm{j}}$ is the final DICL concentration $\left(\mathrm{mg} \mathrm{L}^{-1}\right), \mathrm{V}$ is the volume of the solution $(\mathrm{L})$ and $\mathrm{m}$ is the mass of the adsorbent $(\mathrm{g})$. DICL concentrations were measured using the UV-VIS spectrometer Specord 200 (Analytic Jena, Jena, Germany) at wavelength $278 \mathrm{~nm}$ after filtration of the solution through a $0.45-\mu \mathrm{m}$ syringe filter. All the experiments and measurements were run at $20^{\circ} \mathrm{C}$.

\section{Results and Discussion}

The chemical modification of the initial mesoporous carbon CMK-3 was achieved by three routes, as schematically presented in Figure 1. Two of them are based on an impregnation/carbonization-based route. Initially, CMK-3 was impregnated with the N-rich precursor (dicyandiamide) or N-/S-rich one (thiourea), followed by pyrolysis under inert gas atmosphere. These modification procedures have been recently reported by us to modify activated carbon fiber textiles which are typical microporous material [30,31]. As we showed, the properties of the modified microporous textiles were significantly altered. The choice of dicyandiamide and thiourea as modifiers was made due to their rich content of nitrogen and/or sulfur; at least some fraction of those heteroatoms is expected to be incorporated in the CMK-3 framework.

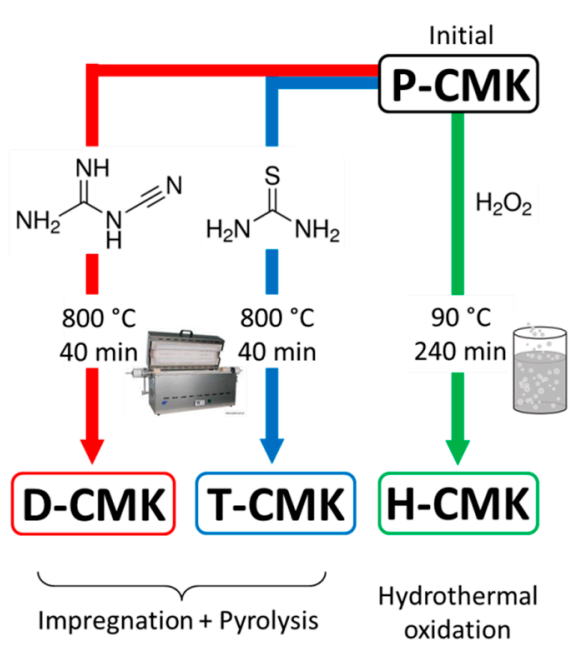

Figure 1. Scheme presenting three modification protocols used in this work.

The third obtained sample was received following a wet oxidation treatment with $\mathrm{H}_{2} \mathrm{O}_{2}$. The former two treatments were previously applied to the nanoporous carbon textiles [30,31], while the third one is a classic wet-oxidation modification process.

The morphology of the initial and modified OMCs was evaluated by SEM (Figure 2 and Figure S2). The images reveal a typical 'sausage-like' structure characteristic of OMC materials [10], i.e., micrometer-size bundles of aggregated rods. This morphology is also typical of SBA-15 template [32], which confirmed a good SBA-15 to CMK-3 replication process. The morphologies of the modified OMC materials resembled each other and also that of initial P-CMK material, which means that the morphological changes caused by modifications were negligible.

The changes on the structural features were examined using nitrogen sorption measurements. The isotherm of all the samples (Figure 3a) had a typical shape for CMK-3 materials and can be classified as that of type IVa, according to the IUPAC (International Union of Pure and Applied Chemistry) classification [33]. The capillary condensation step occurred at relative pressures of about 0.40 , which corresponded to the primary mesopore size of about $4 \mathrm{~nm}$. The corresponding pore size distributions (Figure $3 \mathrm{~b}$ ) revealed two dominant pore fractions for all the samples with the maxima centered at $\sim 1.0 \mathrm{~nm}$ and $\sim 3.1 \mathrm{~nm}$. Only in the case of the sample H-CMK this maximum was shifted towards 
larger pore size of $\sim 3.4 \mathrm{~nm}$, testifying to significant pore enlargement in the course of $\mathrm{H}_{2} \mathrm{O}_{2}$ oxidation. There were notable differences of micropore and mesopore volumes between the samples (Table 1 ). Dicyandiamide treatment (sample D-CMK) resulted in higher micropore and mesopore volumes when compared to the initial sample (P-CMK), while treatment with thiourea (T-CMK) did not change the porous structure significantly. On the contrary, $\mathrm{H}_{2} \mathrm{O}_{2}$ treatment (H-CMK) led to a drastically lowering in volume of both micro- and mesopores. The same trend was observed with relation to $\mathrm{S}_{\mathrm{BET}}$ (specific surface area estimated according to Brunauer, Emmett and Teller method) values. Thus, the proposed thermochemical treatments with the use of dicyandiamide and thiourea as modification agents resulted in better preservation of porosity, while traditional wet oxidation process evidently destroyed significant parts of the porous structure.
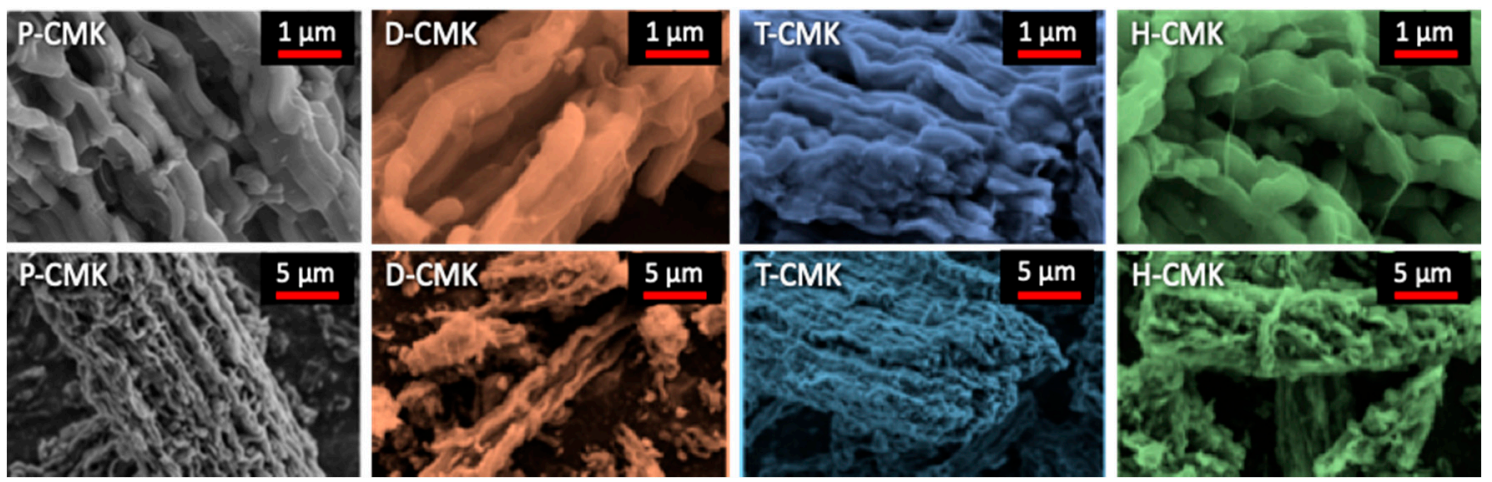

Figure 2. SEM microphotographs of initial and modified OMCs.
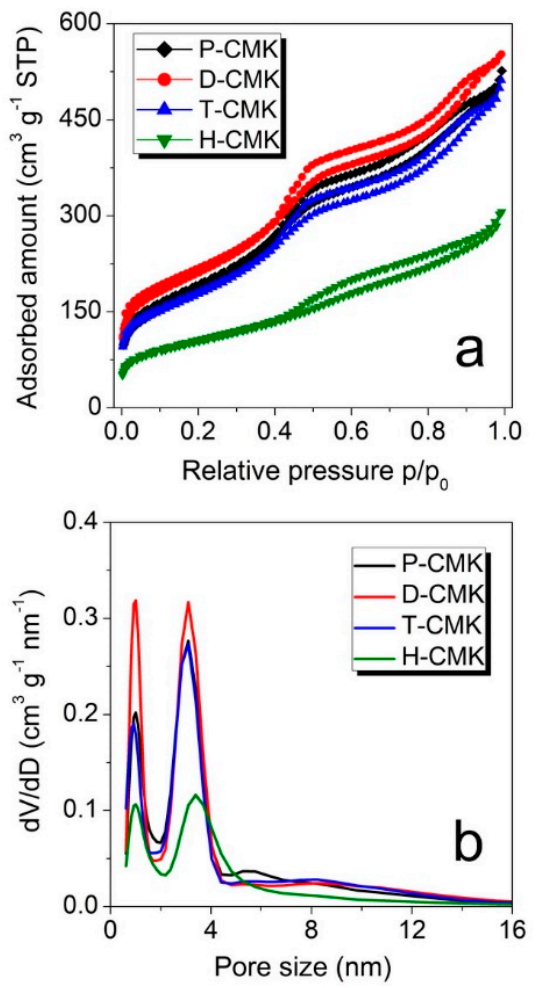

Figure 3. Nitrogen sorption isotherms (a) and corresponding pore size distributions (b) of the studied carbons.

$\mathrm{XRD}$ analysis (Figure S3) revealed the presence of three reflections located at $2 \theta$ angles $\sim 1.0^{\circ}, 1.7^{\circ}$, and $2.0^{\circ}$, respectively, for all the samples apart from H-CMK. These XRD signals were indexed as (10), (11), and (20) reflections, and corresponded to an ordered mesoporous structure with a 2D-hexagonal 
p6mm symmetry [34]. In the case of the H-CMK carbon, only (10) XRD reflection at $2 \theta$ angle $\sim 1.0^{\circ}$ was observed. This observation is in accordance with porosity results showing significant deterioration of the porous structure.

Table 1. Results derived from the nitrogen sorption, $\zeta$ potential, Raman, XPS measurements, and $\mathrm{pH}$ measurements.

\begin{tabular}{|c|c|c|c|c|c|c|c|c|c|c|}
\hline Sample & $\begin{array}{c}S_{\mathrm{BET}} \\
\left(\mathrm{m}^{2} \mathrm{~g}^{-1}\right)\end{array}$ & $\begin{array}{c}V_{\text {mic }} \\
\left(\mathrm{cm}^{3} \mathrm{~g}^{-1}\right)\end{array}$ & $\begin{array}{c}V_{\text {mes }} \\
\left(\mathrm{cm}^{3} \mathrm{~g}^{-1}\right)\end{array}$ & $\begin{array}{c}V_{\text {tot }} \\
\left(\mathrm{cm}^{3} \mathrm{~g}^{-1}\right)\end{array}$ & $\begin{array}{c}\text { Potential } \\
\zeta(\mathrm{mV})\end{array}$ & $\mathbf{I}_{\mathrm{D}} / \mathbf{I}_{G}$ & $\begin{array}{c}\mathrm{C}_{\mathrm{sp} 3} / \mathrm{C}_{\mathrm{sp} 2} \\
\text { Ratio }\end{array}$ & $\begin{array}{c}\text { Initial } \\
\mathrm{pH}\end{array}$ & $\begin{array}{l}\text { Final } \\
\mathrm{pH}\end{array}$ & $\mathrm{pH}_{\text {IEP }}$ \\
\hline P-CMK & 663 & 0.167 & 0.567 & 0.734 & -23.3 & 0.90 & 0.10 & 4.5 & 7.0 & 4.5 \\
\hline D-CMK & 753 & 0.201 & 0.594 & 0.795 & 19.0 & 0.94 & 0.11 & 7.8 & 8.5 & 8.0 \\
\hline T-CMK & 636 & 0.155 & 0.555 & 0.710 & -2.4 & 0.98 & 0.17 & 8.3 & 9.2 & 8.4 \\
\hline H-CMK & 375 & 0.097 & 0.321 & 0.418 & -28.6 & 0.89 & 0.18 & 3.0 & 5.5 & 3.1 \\
\hline
\end{tabular}

Raman spectra (Figure S4) showed two bands typically recognized for graphitized carbon materials: D band at $~ 1350-1358 \mathrm{~cm}^{-1}$ and $\mathrm{G}$ band at $\sim 1591-1595 \mathrm{~cm}^{-1}$. The $\mathrm{G}$ band was assigned to the vibration of $\mathrm{sp}^{2}$-type carbon atoms in a hexagonal lattice, while the $\mathrm{D}$ band was related to the defects and disorders in structures of carbon materials. The relative intensity ratio of $D$ to $G$ band $\left(\mathrm{I}_{\mathrm{D}} / \mathrm{I}_{\mathrm{G}}\right)$ is considered to reflect the graphitization degree of the materials [27], however, it should be interpreted with caution. The CMK-3 carbons were only slightly graphitized and should be considered as $\mathrm{sp}^{2}$-bonded carbons composed mainly of large polycyclic aromatic molecules rather than well-aligned graphitic domains [35]. Graphitization requires temperatures exceeding $2500{ }^{\circ} \mathrm{C}$ or sometimes lower $\left(\sim 1000{ }^{\circ} \mathrm{C}\right)$ but only with the presence of metal-based catalysts enforcing local graphitization [36,37]. It was demonstrated that the apparent G-band may result from two overlapping signals: a proper G-band but also $\mathrm{D}^{\prime}$ band [38]. $\mathrm{D}^{\prime}$ band is a disorder-induced band centered at $1620 \mathrm{~cm}^{-1}$ which is frequently observed in the Raman spectra of disordered graphitic materials like graphene oxide but also for amorphous carbons [39]. The $\mathrm{I}_{\mathrm{D}} / \mathrm{I}_{\mathrm{G}-a p p a r e n t}$ ratios (Table 1) indicate that the oxidation process of P-CMK carbon had little effect on the $\mathrm{I}_{\mathrm{D}} / \mathrm{I}_{\mathrm{G}}$ value. In the case of D-CMK and T-CMK carbons obtained by thermochemical modification of pristine CMK-3, the $\mathrm{I}_{\mathrm{D}} / \mathrm{I}_{\mathrm{G}}$ value increased (especially in the case of $\mathrm{T}-\mathrm{CMK}$ ), thus their graphitization degree was lower in comparison to P-CMK material. Surprisingly, the $\mathrm{I}_{\mathrm{D}} / \mathrm{I}_{\mathrm{G} \text {-apparent }}$ ratio of the $\mathrm{H}-\mathrm{CMK}$ carbon is almost the same as in the case of the pristine P-CMK carbon, which may result from the increase of the relative share of $\mathrm{D}^{\prime}$ band in the apparent $\mathrm{G}$ band. Nevertheless, the $\mathrm{I}_{\mathrm{D}} / \mathrm{I}_{\mathrm{G} \text {-apparent }}$ values were relatively close to each other for all the carbons studied.

Additionally, the surface chemical properties of the samples were also thoroughly investigated. In Figure S5 the values of zeta potential for all the studied samples as a function of solution $\mathrm{pH}$ are presented. All curves intersect with $\mathrm{pH}$ axis at different $\mathrm{pH}$ values. These $\mathrm{pH}$ values are corresponding to the zero value of zeta potential and they are known as isoelectric points $\left(\mathrm{pH}_{\text {IEP }}\right)$. Values of $\mathrm{pH}_{\text {IEP }}$ clearly differed between individual samples: P-CMK and $\mathrm{H}-\mathrm{CMK}$ had $\mathrm{pH}_{\mathrm{IEP}}$ values below 5 (4.5 and 3.1, respectively), most probably related to an abundance of surface oxygen groups. Obviously, after the $\mathrm{H}_{2} \mathrm{O}_{2}$ treatment, the number or/and character of those groups changed, which implies the noticeable shift of $\mathrm{pH}_{\mathrm{IEP}}$ towards lower value. In the case of samples T-CMK and D-CMK, the value of $\mathrm{pH}_{\text {IEP }}$ was high (8.4 and 8.0, respectively), indicating serious changes in surface chemistry of that samples as a result of thermochemical modification with dicyandiamide or thiourea.

The values of potential zeta can be at least partially related with the protonation/deprotonation equilibria of the oxygen functional groups on the carbon surface. For example, two basic carbons (D-CMK and T-CMK) had no carboxyl surface groups (cf. Table S1-O 1s deconvolution) which were considered strongly acidic:

$$
\text { carbon-COOH } \Longleftrightarrow \text { carbon- } \mathrm{COO}^{-}+\mathrm{H}^{+}\left(3 \leq \mathrm{pK}_{\mathrm{a}} \leq 6\right),
$$


while they had more hydroxyl groups (cf. Table S1—O 1s deconvolution), which were weakly acidic:

$$
\text { carbon-OH } \Longleftrightarrow \text { carbon- }-\mathrm{O}^{-}+\mathrm{H}^{+}\left(8 \leq \mathrm{pK}_{\mathrm{a}} \leq 11\right) \text {. }
$$

To look more closely at the changes in surface chemistry heterogeneity as a result of the followed modifications, the superficial chemistry was inspected also by two quantitative methods: XPS and EDS (Energy-Dispersive X-Ray Spectroscopy). The first one is considered a surface-sensitive technique enabling determination of the surface composition to a depth up to several $\mathrm{nm}$ [40], while the second, up to several $\mu \mathrm{m}$ [41]. The first interesting conclusion was that the results collected using both techniques were nearly compatible (Table 2), albeit with two important exceptions, which will be discussed later in detail. XPS analysis reveals that the carbon content for all samples but H-CMK was higher than $91 \%$. Highest carbon content for D-CMK carbon $(93.9 \%)$ resulted most probably from deposition/incorporation of dicyandiamide fragments formed in the course of its thermal decomposition. This effect can be linked with the further development of the porous structure during dicyandiamide modification (cf. Table 1). In the case of T-CMK, the carbon content was almost unchanged, while significant decrease of carbon content to $83.7 \%$ was observed after wet oxidation using $\mathrm{H}_{2} \mathrm{O}_{2}$. In the same time, the oxygen content for $\mathrm{H}-\mathrm{CMK}$ increased up to $15.9 \%$, indicating the oxygen-rich surface chemistry of this sample. In contrast, thermochemical treatment with dicyandiamide and thiourea resulted in decreased oxygen content ( $5.2 \%$ and $4.5 \%$, respectively) when compared with the initial P-CMK carbon (7.0\%). A similar trend was observed in the case of porous carbon textiles modified with dicyandiamide and thiourea in the same way [31]. The presence of nitrogen was not evidenced by XPS for the D-CMK carbon, however, nitrogen was detected by EDS elemental analysis (1.1\%). The most plausible explanation can be that the nitrogen moieties were located in the interior parts of the D-CMK structure, which was inaccessible for XPS due to the scanning depth limited to several $\mathrm{nm}$. Noticeable relative differences of $\mathrm{N}$ and $\mathrm{S}$ contents by two techniques again should be attributed to the specific locations of the heteroatoms in the interior carbon matrix, thus XPS-inaccessible parts of carbon framework.

Table 2. Elemental analysis of the samples studied as given by EDS and XPS methods.

\begin{tabular}{ccccccccccc}
\hline & \multicolumn{4}{c}{ EDS Elemental Analysis * } & \multicolumn{4}{c}{ XPS Elemental Analysis ** } \\
\cline { 2 - 11 } Sample & $\mathbf{C}$ & $\mathbf{O}$ & $\mathbf{N}$ & $\mathbf{S}$ & $\mathbf{C l}$ & $\mathbf{C}$ & $\mathbf{O}$ & $\mathbf{N}$ & $\mathbf{S}$ & $\mathbf{C l}$ \\
& wt.\% & wt.\% & wt.\% & wt.\% & wt.\% & wt.\% & wt.\% & wt.\% & wt.\% & wt.\% \\
\hline P-CMK & 91.0 & 7.9 & 0.0 & 0.4 & 0.1 & 91.0 & 7.0 & 0.0 & 0.0 & 2.0 \\
D-CMK & 93.9 & 4.3 & 1.1 & 0.1 & 0.0 & 94.8 & 5.2 & 0.0 & 0.0 & 0.0 \\
T-CMK & 91.1 & 6.9 & 0.8 & 0.8 & 0.0 & 92.4 & 4.5 & 1.7 & 1.4 & 0.0 \\
H-CMK & 83.7 & 15.0 & 0.0 & 0.1 & 0.3 & 81.8 & 15.9 & 0.0 & 0.0 & 0.8 \\
\hline
\end{tabular}

* EDS elemental analysis averaged from six experimental points. Elements like $\mathrm{Na}, \mathrm{Si}, \mathrm{K}$, and $\mathrm{Ca}$ were also detected (less than $1 \%$ and they were not taken into consideration on the wt. \% expression). ${ }^{* *}$ Elements like Ca or Si were also detected (less than $1 \%$ ).

Deconvolution of the $\mathrm{C} 1 \mathrm{~s}$ and $\mathrm{O} 1 \mathrm{~s}$ core energy levels along with survey spectra is provided in Supplementary Materials (Figures S6-S8), while its graphical representation is shown in Figure 4. The most intensive component after deconvolution of C1s core energy level located at 283.9-284.2 eV was assigned to $\mathrm{sp}^{2}$-bonded $(\mathrm{C}=\mathrm{C})$ carbon structures, which were rather large polycyclic fragments than graphitic domains. The relative contribution of this signal was $\sim 90 \%$ for P-CMK and D-CMK-3 carbons and $\sim 85 \%$ for T-CMK and $\mathrm{H}-\mathrm{CMK}$ carbons. Interestingly, T-CMK had elevated contribution of $\mathrm{sp}^{3}$-bonded $(\mathrm{C}-\mathrm{C})$ carbon fragments, which suggested partial cleavage of double $\mathrm{C}=\mathrm{C}$ bonds, however without significant incorporation of oxygen species. The deconvolution of $\mathrm{C} 1 \mathrm{~s}$ signal shows that a wide range of different functionalities was present on the carbon surface, typical for carbon-based materials, although with different concentrations. The most striking feature of H-CMK was its high content of 
strongly acidic carboxyl groups (6.0\%), while for the rest of the samples this content was significantly lower $(0.0 \%$ for P-CMK, $1.8 \%$ for D-CMK, and $2.1 \%$ for T-CMK).
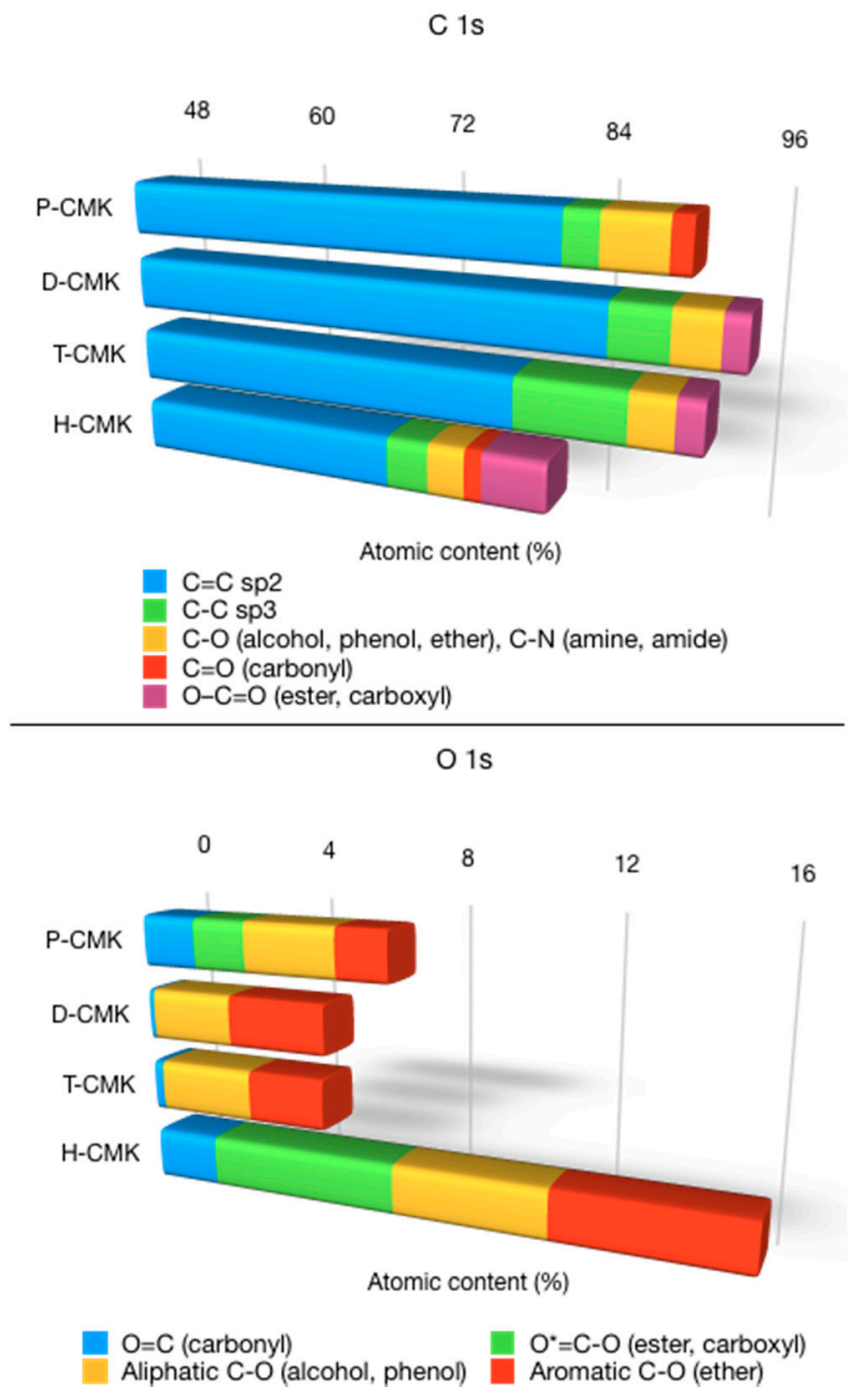

Figure 4. The results of the deconvolution of the XPS spectra of $\mathrm{C} 1 \mathrm{~s}$ and $\mathrm{O} 1 \mathrm{~s}$ core energy levels.

The deconvolution of O1s core energy level resulted in four signals for P-CMK and H-CMK and three signals for D-CMK and T-CMK (Figure S8). Their presence testifies to the abundance of different oxygen species on the surface, albeit with different relative shares [30]. The two most important observations coming from the analysis of deconvoluted O 1s core energy level were: (1) H-CMK exhibited significant contributions of all types of oxygen species, with the most prominent presence of strongly acidic carboxy groups, and (2) D-CMK and T-CMK had no strongly acidic carboxy groups but contained almost entirely weakly acidic phenols and basic ether groups.

In the case of T-CMK carbon, the deconvolution of N 1s and S 2p's core energy levels resulted in the appearance of three or two signals, respectively. The most intensive component of $\mathrm{N} 1 \mathrm{~s}$ core energy level located at $398.7 \mathrm{eV}$ was assigned to pyridinic nitrogen and/or imine [30]. Two other components of $\mathrm{N} 1 \mathrm{~s}$ core energy level located at $400.1 \mathrm{eV}$ and $401.5 \mathrm{eV}$ corresponded to pyrrolic nitrogen/amine and quaternary nitrogen, respectively. Both pyridinic and pyrrolic nitrogen species can be located at the edges of graphene planes and quaternary nitrogen can be located inside graphene layer. Aromatic $\mathrm{sp}^{2}$-hybridized nitrogen, such as in pyridine, and quaternary nitrogen led to substantial electron deficiency in the aromatic ring, which provided a positive charge on the graphene surface. On the 
other hand, the S 2p's core energy level components located at $164.2 \mathrm{eV}\left(\mathrm{S} 2 \mathrm{p}_{3 / 2}\right)$ and $165.4 \mathrm{eV}\left(\mathrm{S} 2 \mathrm{p}_{1 / 2}\right)$ corresponded to thiols, bisulfides, and $\mathrm{R}_{2}-\mathrm{SO}$ groups, which can be located at the edges of graphene layers [30].

Those considerations were confirmed by observing the initial $\mathrm{pH}$ values (see Table 1 ) of the solution containing carbons immersed in water and stabilized overnight. It can be seen that the initial $\mathrm{pH}$ of CMK-P carbon was 4.5, indicating an abundance of acidic oxygen groups (e.g., carboxyl), which hydrolyze releasing $\mathrm{H}_{3} \mathrm{O}^{+}$ions, resulting in lowered $\mathrm{pH}$ value. $\mathrm{H}_{2} \mathrm{O}_{2}$ oxidation resulted in an even more acidic surface $(\mathrm{pH}=3.0)$ that suggests remarkable increase of the oxygen acidic groups on the surface, which was clearly confirmed by the deconvolution of $\mathrm{O} 1$ s core energy level. In contrast, the initial $\mathrm{pH}$ of both thermochemically modified carbons was basic (i.e., 7.8 for D-CMK and 8.3 for T-CMK), indicating basic surface functionalities. Again, this was in total agreement with the deconvoluted $\mathrm{O} 1 \mathrm{~s}$ core energy level, where abundancies of basic ether groups were present in the case of those thermochemically treated carbons. For better clarity in Figure S9 content of functional group versus $\mathrm{pH}_{\mathrm{IEP}}$ of the carbons studied was presented.

It should be noted at this point that the acidity/basicity balance of carbons was not only related to the presence of more or less acidic surface oxygen functionalities but also to the delocalized $\pi$-electrons in polyaromatic/graphene domains, which had a basic character, i.e., protons were hydrated according to $\mathrm{C}_{\pi}+2 \mathrm{H}_{2} \mathrm{O} \rightarrow \mathrm{C}_{\pi} \mathrm{H}_{3} \mathrm{O}^{+}+\mathrm{OH}^{-}$[42]. Thus, the analyzed surface basicity was not straightforward because it was not entirely associated with surface oxygen complexes. Three general observations derived from the literature can be shortly summarized as following [42]: (1) Acidic sites were associated with the surface oxygen complexes, although some of them were less acidic (e.g., phenols) while others were more acidic (e.g., carboxyls) [43]; (2) basic sites were concentrations of delocalized $\pi$-electrons within the polyaromatic/graphene domains of the carbon structure; and (3) the increase of the nitrogen content of the polyaromatic/graphene domains enhanced remarkably the basicity. Considering all those aspects, it can be concluded that the basicity of the D-CMK and T-CMK carbons resulted from both simultaneously occurring factors: The increasing content of delocalized $\pi$-electrons and decreasing content of the acidic oxygen groups, during both thermochemical treatments, oxygen groups were stripped from the surface. Since dicyandiamide and thiourea molecules do not contain oxygen, the stripped oxygen species cannot be replaced by other oxygen species. As a result, thermochemical activation led to materials with increased carbon content and decreased oxygen content. It was demonstrated before in the literature that the surface basic sites of carbons are mainly of the Lewis type [44]. Additionally, incorporation of basic nitrogen groups also remarkably contributed to the increased basicity. In the case of $\mathrm{H}_{2} \mathrm{O}_{2}$ oxidation, the opposite processes occurred: large amount of acidic oxygen groups were generated which localized $\pi$-electrons, thus suppressing the effect of the $\pi$-electrons responsible for carbon basicity. XPS revealed that carbon content dropped from $91.0 \%$ to $81.8 \%$ during $\mathrm{H}_{2} \mathrm{O}_{2}$ oxidation with a simultaneous increase of oxygen from $7.0 \%$ to $15.9 \%$, which clearly supported those considerations.

Well-developed porosity and rich surface chemistry make the obtained materials good candidates to be used as sorbents. Two main reasons make the obtained carbons potentially useful for drug removal: (1) a mesoporous structure providing fast transport of bulky drug molecules by diffusion through the mesopores towards the adsorption centers, and (2) tunable surface chemistry, which can be used to tailor the strength of interactions between adsorbates and the carbon surface. To verify this hypothesis and if it is applicable for pharmaceuticals' removal, and specifically of diclofenac, adsorption experiments were conducted. On the basis of our previous studies $[34,45]$ as well as the literature review (see Table S2), the $\mathrm{pH}$ value was set as 5.5-6.0. Around $\mathrm{pH} \approx 4$, the formation of not-dissociated acidic and minimally soluble form of diclofenac took place ( $\mathrm{pK} \mathrm{a}_{\mathrm{a}}$ value of DICL was 4.15 [34]) which made the interpretation of the adsorption results erroneous due to DICL precipitation. However, at $\mathrm{pH}>5.5$, the soluble ionized form of DICL was almost exclusively present in the solution, which made it possible to run adsorption experiments using unbuffered solutions of $\mathrm{pH} \approx 5.5$. 
Figure 5a collects the adsorption kinetics of DICL for the herein studied samples. As shown, the adsorption kinetics were relatively fast for carbon materials, mainly due to the open, interconnected three-dimensional pore structures replicated from the SBA- 15 template. After $5 \mathrm{~h}$, all the samples but P-CMK had uptakes close to equilibrium values $(92 \%, 88 \%$, and $95 \%$, for D-CMK, T-CMK, and H-CMK, respectively). In the case of initial P-CMK carbon, only $77 \%$ of equilibrium value was adsorbed after that time. This behavior of P-CMK sample can be explained by its low wettability due to few oxygen groups on the surface. Although the total oxygen content was high ( $7.0 \%$ by XPS), most of those groups were located in the micropores but not in larger trafficking pores; thus, the migration of the DICL ions to the adsorption was slowed down. It is worth noting that the porous structure of the sample $\mathrm{H}-\mathrm{CMK}$ was predominantly mesoporous; thus, the adsorption process was fast and was additionally facilitated by the abundance of surface oxygen groups introduced in the course of $\mathrm{H}_{2} \mathrm{O}_{2}$ oxidation. In the case of classical microporous activated carbon, Norit SX2 was used for comparative purposes, and the adsorption was significantly slower and the equilibrium was reached after 8 days (basic characterization of Norit SX2 and its adsorption performance is presented in Figures S10 and S11). This was due to the closed and tortuous microporous structure without a significant fraction of larger pores, which would act as transporting/diffusion channels for DICL delivering to adsorption centers located in micropores.
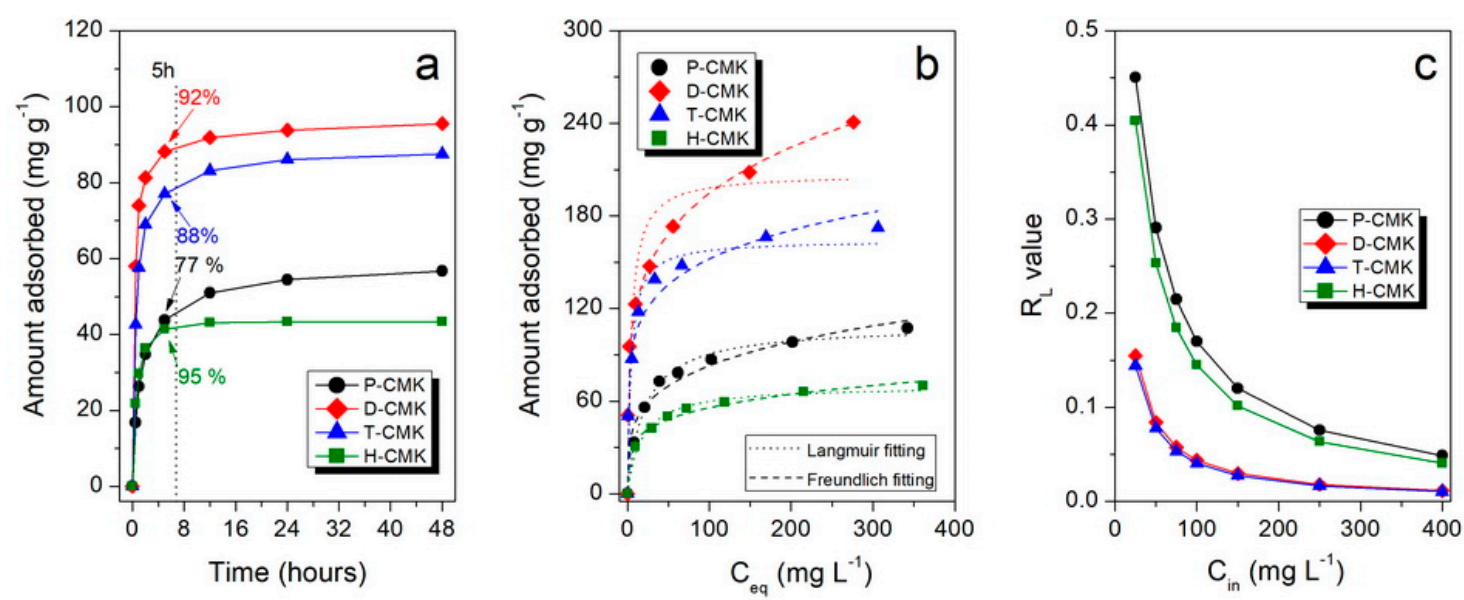

Figure 5. (a) DICL adsorption kinetics onto the studied CMK materials (initial concentration of DICL: $50 \mathrm{mg} \mathrm{L}^{-1}$ ), (b) adsorption isotherms along with their fitting using Langmuir and Freundlich models, (c) values of separation factor $\left(R_{L}\right)$ for different DICL initial concentrations used in this study.

Adsorption isotherms, presenting the dependence of the adsorbed amount of DICL on its equilibrium concentration, are shown in Figure 5b. The highest sorption capacities (SCs) were observed in thermochemically modified samples, D-CMK and T-CMK (241 and $172 \mathrm{mg} \mathrm{g}^{-1}$, respectively), while for the initial P-CMK and $\mathrm{H}_{2} \mathrm{O}_{2}$-treated $\mathrm{H}$-CMK samples those values were significantly lower (107 and $70 \mathrm{mg} \mathrm{g}^{-1}$, respectively). Another interesting observation is that D-CMK and T-CMK carbons can adsorb as much as $99 \%$ and $98 \%$ of DICL, respectively, from the solution with its lowest concentration tested (i.e., $25 \mathrm{mg} \mathrm{L}^{-1}$ ). This means that almost quantitative adsorption of DICL was provided by those materials, while for P-CMK and H-CMK, corresponding uptakes were much lower: $67 \%$ and $61 \%$, respectively. The key role of positive influence on adsorptive capability upon surface chemistry modification can be derived by the comparison of the sorption capacities per $\mathrm{S}_{\mathrm{BET}}$ (Table 3). All modifications led to an increase of the surface uptake capability, although the positive impact compared to P-CMK was the lowest for H-CMK $(+16 \%)$ and the highest for D-CMK $(+\sim 100 \%)$.

The sorption equilibrium data were modeled with Langmuir and Freundlich adsorption models (the appropriate equations can be found in our previous works $[46,47]$ ), and the fitted results are presented in Figure $5 b$ while the fitting parameters and determination coefficients, $R^{2}$, in Table 3 . The fitting clearly shows that for all the samples, except D-CMK, the Langmuir models assuming the 
formation of adsorbed monolayer provides fit better, and that is reflected in $\mathrm{R}^{2}$ values. Interestingly, only in the case of the D-CMK was the Freundlich model significantly better (what can be easily seen by comparing the fitted Freundlich and Langmuir curves).

Table 3. Sorption capacities (SC) of DICL and parameters calculated from the Langmuir and Freundlich fitting.

\begin{tabular}{|c|c|c|c|c|c|c|c|c|c|c|}
\hline \multirow{2}{*}{ Sample } & \multirow{2}{*}{$\begin{array}{c}\mathrm{SC} \\
\left(\mathrm{mg} \mathrm{g}^{-1}\right)\end{array}$} & \multirow{2}{*}{$\begin{array}{c}\mathrm{SC} / \mathrm{S}_{\mathrm{BET}} \\
\left(\mathrm{mg} \mathrm{m}^{-2}\right)\end{array}$} & \multicolumn{4}{|c|}{ Langmuir Fitting } & \multicolumn{3}{|c|}{ Freundlich Fitting } & \multirow{2}{*}{$\begin{array}{c}\text { RDE } \\
(\%)\end{array}$} \\
\hline & & & $\mathrm{q}_{\mathrm{m}}$ & $\mathrm{K}_{\mathrm{L}}$ & $\mathbf{R}^{2}$ & $R_{L(400)}$ & $\mathrm{K}_{\mathrm{F}}$ & $\mathbf{n}$ & $R^{2}$ & \\
\hline P-CMK & 107 & 0.161 & 109 & 0.05 & 0.993 & 0.05 & 27 & 0.25 & 0.924 & 28.5 \\
\hline D-CMK & 241 & 0.320 & 207 & 0.22 & 0.869 & 0.01 & 75 & 0.21 & 0.994 & 14.3 \\
\hline T-CMK & 172 & 0.270 & 164 & 0.24 & 0.941 & 0.01 & 71 & 0.17 & 0.937 & 38.6 \\
\hline $\mathrm{H}-\mathrm{CMK}$ & 70 & 0.187 & 70 & 0.06 & 0.983 & 0.04 & 21 & 0.21 & 0.958 & 92.4 \\
\hline
\end{tabular}

Explanation of the above-mentioned phenomenon is strictly linked to the mechanisms governing the adsorption of DICL. Diclofenac has been reported to interact with carbonaceous surfaces via three types of interactions: (1) van der Waals electrostatic forces, (2) hydrogen-bonding formation, and (3) noncovalent $\pi-\pi$ stacking interactions between the aromatic rings. In the case of the sample D-CMK nonspecific electrostatic interaction between the surface and DICL anions was particularly strong because of the positively charged surface (supported by the positive value of $\zeta$ potential). In contrast, T-CMK surface charge was almost neutral; thus, electrostatic interactions were not the favorable driving force for DICL adsorption. Most probably, specific interactions between DICL anion and N- and Ssurface groups were partially responsible for high DICL uptake, although it cannot be fully supported on the basis of the collected data.

In Table 2, the $\mathrm{pH}$ changes of DICL solution before and after the adsorption process are collected. In the case of both thermochemically modified carbons, the change of solution $\mathrm{pH}$ towards higher values can be explained by the participation of $\mathrm{H}_{3} \mathrm{O}^{+}$cations from the solution into the formation of hydrogen bonds between DICL anions and carbon surface. Thus, the solution pH after DICL adsorption was at high level. An even stronger effect was observed in the case of P-CMK and H-CMK-3 carbons (increase of $\mathrm{pH}$ by 2.5 units).

The lowest uptakes of DICL onto P-CMK and H-CMK can be related to the strong electrostatic repulsion between the negatively charged surface and DICL anions, as well as to a preferable monolayered adsorption via the carboxylic groups that prevent a second adsorption layer, as above mentioned. In the case of the H-CMK, surface area $\left(375 \mathrm{~m}^{2} \mathrm{~g}^{-1}\right)$ and volume of micropores $\left(0.097 \mathrm{~cm}^{3} \mathrm{~g}^{-1}\right)$ were the lowest among the samples studied, and so this can be the reason behind the lowest DICL uptake (only $70 \mathrm{mg} \mathrm{g}^{-1}$ ) compared to the rest of the samples. Clearly, the type of functional groups present on the surface and porous structure were the two most important and intercorrelated parameters affecting the sorption efficiency. Well-developed micro- and mesoporous structure without proper functionalization cannot provide fast and high adsorption, which was clearly seen for the P-CMK sample: Lack of polar surface slowed down the adsorption kinetics due to low wettability, but also resulted in insufficiently strong interaction of DICL with the adsorption centers.

Knowing the value of the Langmuir equilibrium constant (related to the adsorption energy), $\mathrm{K}_{\mathrm{L}}$, allows us to calculate so-called separation factor, $\mathrm{R}_{\mathrm{L}}$, which is a dimensionless constant accounting for affinity between the sorbate and sorbent [34]. In general, $\mathrm{R}_{\mathrm{L}}=1$ corresponds to the linear adsorption isotherm, when $R_{\mathrm{L}}<<1$ the adsorption is highly favorable (in the extreme but theoretical case where $\mathrm{R}_{\mathrm{L}}$ $=0$, adsorption is considered irreversible). $R_{L}$ values for different initial concentrations are presented in Figure $5 \mathrm{c}$ and for the highest initial concentration used $\left(400 \mathrm{mg} \mathrm{L}^{-1}\right)$ also in Table 3 . In the case of $\mathrm{D}-\mathrm{CMK}$ and T-CMK, the $\mathrm{R}_{\mathrm{L}}$ values are lower than the corresponding values of P-CMK and H-CMK for any initial concentration, indicating high adsorption process.

To describe in more detail the interactions between DICL and the studied materials, desorption tests were run. A 0.9 wt. \% solution of $\mathrm{NaCl}$ was used as DICL desorption medium from the loaded 
samples. Its desorption efficiency was confirmed by us previously in the case of silica materials, where an increase of the ionic strength resulted in significant desorption of DICL [48,49]. The relative desorption efficiencies (RDE) are given in Table 3. It can be seen that there were remarkable differences between the samples: Low RDE was observed for D-CMK (14.3\%), moderate RDE for P-CMK and T-CMK (28.5 and 38.6, respectively), and almost total desorption for H-CMK (92.4\%). The observed differences can be explained by distinct alterations of surface chemistry and porosity of the carbon studied. D-CMK had the highest surface charge, as indicated by its positive $\zeta$ potential. At the same time, it had highest volume of micropores, where the bulky DICL ions can be strongly and irreversibly adsorbed. The micropores of CMK-3 carbon are textural slit-like micropores (i.e., they do not result from the templating protocol but are formed on the course of carbonization, similarly to the micropores of activated carbon). Due to the slit-like shape of the pores, even big molecules can enter the pore spaces if only one of the molecular dimensions of the adsorbate is smaller than the pore size, regardless of its two remaining dimensions. The length of diclofenac was approximately $1.25 \mathrm{~nm}$ (taking into account van der Waals' radii of the outermost protons). However, its height was less than $1 \mathrm{~nm}$ so there were no serious steric hindrances which could limit free diffusion of diclofenac into the micropore space [34].

Low RDE of only $14.3 \%$ is most probably associated with the desorption of DICL anions adsorbed in mesopores due to the increase of ionic strength suppressing electrostatic interactions between the positively charged surface and negatively charged DICL ions. However, it is plausible to assume that DICL anions adsorbed in narrow slit-like micropores were bonded via electrostatic interactions, hydrogen bonds, and $\pi-\pi$ stacking, which are difficult to brake due to limited water access to the filled micropores. In the case of the P-CMK and T-CMK, the attractive electrostatic interactions were weaker (particularly for P-CMK) and the volume of micropores was significantly lower than that of D-CMK. Both factors resulted in higher desorption efficiency. In the case of $\mathrm{H}-\mathrm{CMK}$, the surface was negatively charged and the volume of micropores able to provide the strong binding forces was low, so diclofenac could be desorbed almost quantitatively.

The collected results were compared with the current up-to-date papers reporting adsorption of diclofenac by different carbonaceous materials. As it can be seen, most of the studies indicate adsorption mechanism based on $\pi-\pi$ interactions, hydrogen bonding, and electrostatic interactions. The possible interactions are schematically shown on Figure 6.

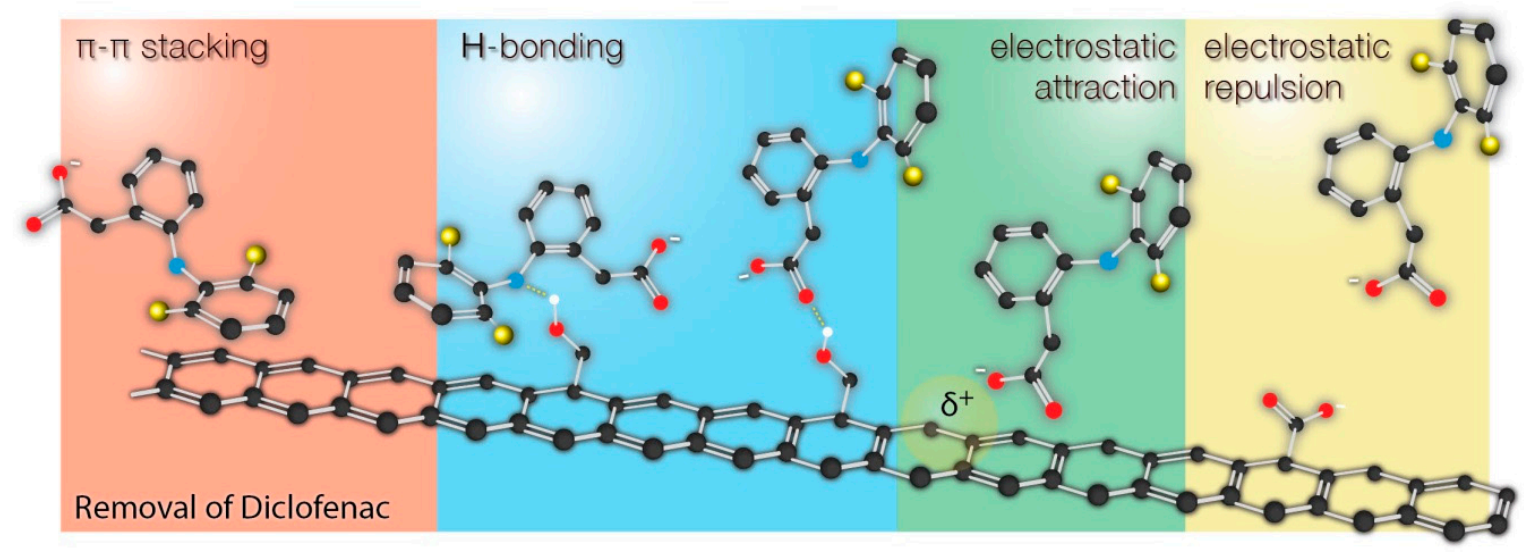

Figure 6. Different adsorption mechanisms involved in the sorption of diclofenac by carbon-based materials.

The possible interactions of diclofenac (as well as the other pharmaceuticals with similar structure) with the surface are shown in Figure 6. It should be clearly underlined here that it is difficult or even impossible to attribute the enhanced sorption of diclofenac to the presence of specific functional groups on the surface. The chemistry of carbon surface is very rich in various chemical groups. All modification protocols (including those described in this work) do not qualitatively transform one 
type of groups into another, but rather change the relative contributions of those groups to the total surface chemistry. Changes of surface chemistry result in the changes of surface charge, $\pi$-basicity (functional groups localize $\pi$ electrons), which results in different adsorption properties. As a result, the changes of surface chemistry affect even more nonspecific interactions $(\pi-\pi$ stacking and electrostatic interactions) than the specific interactions of diclofenac with particular functional groups (hydrogen bonding). Moreover, the adsorption performance was also linked with the changes of porous structure, making the discussion even more complex. For example, due to the significant fraction of microporosity, some surface groups may be accessible for $\mathrm{H}_{3} \mathrm{O}^{+}$ions but not to diclofenac. All these factor make it difficult to propose one strict adsorption mechanism of diclofenac onto carbon-based materials. On the contrary, such a straightforward mechanism can be found in the case of silica-based materials with a similar mesoporous structure but a well-defined surface chemistry $[34,45,50,51]$.

\section{Conclusions}

Different modification routes used in this study resulted in mesoporous CMK-3 carbons with various surface chemical heterogeneity and structural features. Thermochemical modification with dicyandiamide or thiourea resulted in the ordered mesoporous carbons with high basicity caused by the presence of significant amounts of heteroatom-based functional groups but also increased $\mathrm{sp}^{2}$-hybridized carbon content. The XPS elemental analysis revealed a remarkable decrease of the total oxygen content for thermochemically treated carbons with simultaneous increase of the total carbon content (94.8\% for D-CMK and $92.4 \%$ for T-CMK). In contrast, classical wet oxidation resulted in the elevated oxygen content with a predominant fraction of acidic surface groups (mainly phenols and carboxyls). The porous structure was either intact (T-CMK) or altered (D-CMK) during modification. In contrast, classical wet $\mathrm{H}_{2} \mathrm{O}_{2}$ oxidation led to a material with deteriorated porosity and ordering.

The adsorption of diclofenac sodium onto modified mesoporous carbons was strongly dependent on both surface chemistry and porous structure. The dicyandiamide-modified sample (D-CMK) exhibited the highest adsorption capacity $\left(241 \mathrm{mg} \mathrm{g}^{-1}\right)$ and the lowest desorption extent (14.3\%), while the $\mathrm{H}_{2} \mathrm{O}_{2}$-oxidated $\mathrm{H}$-CMK sample had the lowest adsorption capacity $\left(70 \mathrm{mg} \mathrm{g}^{-1}\right)$ and the highest desorption $(92.4 \%)$. The collected results show that the adsorption of pharmaceuticals on carbon-based materials depends on a complex interrelationship between the factors related to surface chemistry and porous structure as well as the wettability of the carbon surface.

Supplementary Materials: The following are available online at http://www.mdpi.com/1996-1944/13/7/1625/s1. Figure S1: Characterization of the SBA-15 template: Nitrogen sorption isotherm (a), XRD diffractogram (b), and SEM images (c,d). Figure S2: SEM microphotographs of the carbons studied. Figure S3: XRD diffractograms of the carbons studied. Figure S4: Raman spectra of the carbons studied. Figure S5: Values of zeta potential of the studied carbons as a function of $\mathrm{pH}$. Figure S6: XPS survey spectra for the carbons studied: P-CMK (a), H-CMK (b), D-CMK (c), T-CMK (d). Figure S7: Deconvolution of C 1s energy level for the carbons studied: P-CMK (a), H-CMK (b), D-CMK (c), T-CMK (d). Figure S8: Deconvolution of O 1s energy level for the carbons studied: P-CMK (a), H-CMK (b), D-CMK (c), T-CMK (d). Figure S9: Functional group content versus $\mathrm{pH}_{\text {IEP }}$ of the carbons studied. Figure S10: Nitrogen adsorption isotherm of Norit SX2 (left), SEM images of Norit SX2 (right). Figure S11. Comparison of DICL adsorption kinetics onto the studied CMK materials and Norit SX2 carbon (initial concentration of DICL: $50 \mathrm{mg} \mathrm{L}^{-1}$ ). Table S1: Results of the deconvolution of the XPS C $1 \mathrm{~s}$ and O 1 s core energy levels. Table S2: Comparison of DICL maximum adsorption capacities by carbon-derived sorbents reported in the literature.

Author Contributions: Conceptualization, R.O., R.D. and M.B.; data curation, R.O., D.A.G., I.A., R.D. and M.B.; formal analysis, R.O.; investigation, R.O., E.Z., R.D. and M.B.; methodology, R.O., E.Z., D.A.G., I.A., R.D. and M.B.; resources, E.Z., R.D. and M.B.; supervision, R.D. and M.B.; validation, M.B.; visualization, R.O., D.A.G. and M.B.; writing—original draft, R.O. and M.B.; writing—review and editing, R.O., D.A.G., I.A., R.D. and M.B. All authors have read and agreed to the published version of the manuscript.

Funding: This research received no external funding.

Acknowledgments: Some instrumental characterization of the materials was carried out with the equipment purchased thanks to the financial support of the European Regional Development Fund in the framework of the Polish Innovation Economy Operational Program (contract no. POIG.02.01.00-06-024/09 Center of Functional Nanomaterials). Konrad Terpiłowski is acknowledged for potential $\zeta$ measurements. 
Conflicts of Interest: The authors declare no conflict of interest.

\section{References}

1. Ryoo, R.; Sang, H.J.; Jun, S. Synthesis of Highly Ordered Carbon Molecular Sieves via Template-Mediated Structural Transformation. J. Phys. Chem. B 1999, 103, 7743-7746. [CrossRef]

2. Ryoo, R.; Joo, S.H.; Kruk, M.; Jaroniec, M. Ordered Mesoporous Carbons. Adv. Mater. 2001, 13, $677-681$. [CrossRef]

3. Meng, Y.; Gu, D.; Zhang, F.; Shi, Y.; Yang, H.; Li, Z.; Yu, C.; Tu, B.; Zhao, D. Ordered Mesoporous Polymers and Homologous Carbon Frameworks: Amphiphilic Surfactant Templating and Direct Transformation. Angew. Chem. Int. Ed. 2005, 44, 7053-7059. [CrossRef] [PubMed]

4. Meng, Y.; Gu, D.; Zhang, F.; Shi, Y.; Cheng, L.; Feng, D.; Wu, Z.; Chen, Z.; Wan, Y.; Stein, A.; et al. A Family of Highly Ordered Mesoporous Polymer Resin and Carbon Structures from Organic-Organic Self-Assembly. Chem. Mater. 2006, 18, 4447-4464. [CrossRef]

5. Castro-Gutiérrez, J.; Sanchez-Sanchez, A.; Ghanbaja, J.; Díez, N.; Sevilla, M.; Celzard, A.; Fierro, V. Synthesis of perfectly ordered mesoporous carbons by water-assisted mechanochemical self-assembly of tannin. Green Chem. 2018, 20, 5123-5132. [CrossRef]

6. Li, L.; Zou, L.; Song, H.; Morris, G. Ordered mesoporous carbons synthesized by a modified sol-gel process for electrosorptive removal of sodium chloride. Carbon 2009, 47, 775-781. [CrossRef]

7. Lee, J.; Kim, J.; Hyeon, T. Recent Progress in the Synthesis of Porous Carbon Materials. Adv. Mater. 2006, 18, 2073-2094. [CrossRef]

8. $\quad$ Liang, C.; Hong, K.; Guiochon, G.A.; Mays, J.W.; Dai, S. Synthesis of a Large-Scale Highly Ordered Porous Carbon Film by Self-Assembly of Block Copolymers. Angew. Chem. Int. Ed. 2004, 43, 5785-5789. [CrossRef]

9. Wu, Z.; Zhao, D. Ordered mesoporous materials as adsorbents. Chem. Commun. 2011, 47, $3332-3338$. [CrossRef]

10. Barczak, M.; Michalak-Zwierz, K.; Gdula, K.; Tyszczuk-Rotko, K.; Dobrowolski, R.; Dabrowski, A. Ordered mesoporous carbons as effective sorbents for removal of heavy metal ions. Microporous Mesoporous Mater. 2015, 211, 162-173. [CrossRef]

11. Antolini, E. Carbon supports for low-temperature fuel cell catalysts. Appl. Catal. B Environ. 2009, 88, 1-24. [CrossRef]

12. Liang, H.-W.; Wei, W.; Wu, Z.-S.; Feng, X.; Müllen, K. Mesoporous Metal-Nitrogen-Doped Carbon Electrocatalysts for Highly Efficient Oxygen Reduction Reaction. J. Am. Chem. Soc. 2013, 135, 16002-16005. [CrossRef] [PubMed]

13. Lin, T.; Chen, I.-W.; Liu, F.; Yang, C.; Bi, H.; Xu, F.; Huang, F. Nitrogen-doped mesoporous carbon of extraordinary capacitance for electrochemical energy storage. Science 2015, 350, 1508-1513. [CrossRef] [PubMed]

14. Xing, W.; Qiao, S.Z.; Ding, R.G.; Li, F.; Lu, G.Q.; Yan, Z.F.; Cheng, H.M. Superior electric double layer capacitors using ordered mesoporous carbons. Carbon 2006, 44, 216-224. [CrossRef]

15. Nishihara, H.; Kyotani, T. Templated Nanocarbons for Energy Storage. Adv. Mater. 2012, 24, 4473-4498. [CrossRef]

16. Karavasili, C.; Amanatiadou, E.P.; Sygellou, L.; Giasafaki, D.K.; Steriotis, T.A.; Charalambopoulou, G.C.; Vizirianakis, I.S.; Fatouros, D.G. Development of new drug delivery system based on ordered mesoporous carbons: Characterisation and cytocompatibility studies. J. Mater. Chem. B 2013, 1, 3167-3174. [CrossRef]

17. Sánchez-Sánchez, Á.; Suárez-García, F.; Martínez-Alonso, A.; Tascón, J.M.D. pH-responsive ordered mesoporous carbons for controlled ibuprofen release. Carbon 2015, 94, 152-159. [CrossRef]

18. Hartmann, M. Ordered Mesoporous Materials for Bioadsorption and Biocatalysis. Chem. Mater. 2005, 17, 4577-4593. [CrossRef]

19. Vinu, A.; Hossian, K.Z.; Srinivasu, P.; Miyahara, M.; Anandan, S.; Gokulakrishnan, N.; Mori, T.; Ariga, K.; Balasubramanian, V.V. Carboxy-mesoporous carbon and its excellent adsorption capability for proteins. J. Mater. Chem. 2007, 17, 1819-1825. [CrossRef]

20. Goscianska, J.; Olejnik, A.; Nowak, I.; Marciniak, M.; Pietrzak, R. Stability analysis of functionalized mesoporous carbon materials in aqueous solution. Chem. Eng. J. 2016, 290, 209-219. [CrossRef] 
21. Benzigar, M.R.; Talapaneni, S.N.; Joseph, S.; Ramadass, K.; Singh, G.; Scaranto, J.; Ravon, U.; Al-Bahily, K.; Vinu, A. Recent advances in functionalized micro and mesoporous carbon materials: Synthesis and applications. Chem. Soc. Rev. 2018, 47, 2680-2721. [CrossRef]

22. Jedynak, K.; Szczepanik, B.; Rędzia, N.; Słomkiewicz, P.; Kolbus, A.; Rogala, P. Ordered Mesoporous Carbons for Adsorption of Paracetamol and Non-Steroidal Anti-Inflammatory Drugs: Ibuprofen and Naproxen from Aqueous Solutions. Water 2019, 11, 1099. [CrossRef]

23. Hubetska, T.; Kobylinska, N.; García, J.R. Efficient adsorption of pharmaceutical drugs from aqueous solution using a mesoporous activated carbon. Adsorption 2020, 26, 251-266. [CrossRef]

24. Ji, L.; Liu, F.; Xu, Z.; Zheng, S.; Zhu, D. Adsorption of Pharmaceutical Antibiotics on Template-Synthesized Ordered Micro- and Mesoporous Carbons. Environ. Sci. Technol. 2010, 44, 3116-3122. [CrossRef]

25. Moreno-Tovar, R.; Terrés, E.; Rangel-Mendez, J.R. Oxidation and EDX elemental mapping characterization of an ordered mesoporous carbon: $\mathrm{Pb}(\mathrm{II})$ and $\mathrm{Cd}(\mathrm{II})$ removal. Appl. Surf. Sci. 2014, 303, 373-380. [CrossRef]

26. Lezanska, M.; Pietrzyk, P.; Sojka, Z. Investigations into the structure of nitrogen-containing CMK-3 and OCM-0.75 carbon replicas and the nature of surface functional groups by spectroscopic and sorption techniques. J. Phys. Chem. C 2010, 114, 1208-1216. [CrossRef]

27. Baikousi, M.; Daikopoulos, C.; Georgiou, Y.; Bourlinos, A.; Zbořil, R.; Deligiannakis, Y.; Karakassides, M.A. Novel Ordered Mesoporous Carbon with Innate Functionalities and Superior Heavy Metal Uptake. J. Phys. Chem. C 2013, 117, 16961-16971. [CrossRef]

28. Ebele, A.J.; Abou-Elwafa Abdallah, M.; Harrad, S. Pharmaceuticals and personal care products (PPCPs) in the freshwater aquatic environment. Emerg. Contam. 2017, 3, 1-16. [CrossRef]

29. Kaldenhoven, R.G.; Hill, J.M. Determining the pore structure of activated carbon by nitrogen gas adsorption. Catalysis 2018, 30, 41-63.

30. de Falco, G.; Barczak, M.; Montagnaro, F.; Bandosz, T.J. A New Generation of Surface Active Carbon Textiles as Reactive Adsorbents of Indoor Formaldehyde. ACS Appl. Mater. Interfaces 2018, 10, 8066-8076. [CrossRef] [PubMed]

31. Barczak, M.; Bandosz, T.J. Evaluation of nitrogen- and sulfur-doped porous carbon textiles as electrode materials for flexible supercapacitors. Electrochim. Acta 2019, 305, 125-136. [CrossRef]

32. Barczak, M.; Dobrowolski, R.; Dobrzyńska, J.; Zięba, E.; Dąbrowski, A. Amorphous and ordered organosilicas functionalized with amine groups as sorbents of platinum (II) ions. Adsorption 2013, 19, 733-744. [CrossRef]

33. Thommes, M.; Kaneko, K.; Neimark, A.V.; Olivier, J.P.; Rodriguez-Reinoso, F.; Rouquerol, J.; Sing, K.S.W. IUPAC Technical Report Physisorption of gases, with special reference to the evaluation of surface area and pore size distribution (IUPAC Technical Report). Pure Appl. Chem. 2015, 87, 1051-1069. [CrossRef]

34. Barczak, M.; Wierzbicka, M.; Borowski, P. Sorption of diclofenac onto functionalized mesoporous silicas: Experimental and theoretical investigations. Microporous Mesoporous Mater. 2018, 264, 254-264. [CrossRef]

35. Inagaki, S.; Yokoo, Y.; Miki, T.; Kubota, Y. Improvement of electric double-layer capacitance of ordered mesoporous carbon CMK-3 by partial graphitization using metal oxide catalysts. Microporous Mesoporous Mater. 2013, 179, 136-143. [CrossRef]

36. Wu, Z.; Li, W.; Xia, Y.; Webley, P.; Zhao, D. Ordered mesoporous graphitized pyrolytic carbon materials: Synthesis, graphitization, and electrochemical properties. J. Mater. Chem. 2012, 22, 8835-8845. [CrossRef]

37. Nettelroth, D.; Schwarz, H.-C.; Burblies, N.; Guschanski, N.; Behrens, P. Catalytic graphitization of ordered mesoporous carbon CMK-3 with iron oxide catalysts: Evaluation of different synthesis pathways. Phys. Status Solidi 2016, 213, 1395-1402. [CrossRef]

38. King, A.A.K.; Davies, B.R.; Noorbehesht, N.; Newman, P.; Church, T.L.; Harris, A.T.; Razal, J.M.; Minett, A.I. A New Raman Metric for the Characterisation of Graphene oxide and its Derivatives. Sci. Rep. 2016, 6, 19491. [CrossRef]

39. Dresselhaus, M.S.; Jorio, A.; Souza Filho, A.G.; Saito, R. Defect characterization in graphene and carbon nanotubes using Raman spectroscopy. Philos. Trans. R. Soc. A 2010, 368, 5355-5377. [CrossRef]

40. Burrell, M.C. Chemical Analysis, Electron Spectroscopy. Encycl. Mater. Sci. Technol. 2001, 1142-1149.

41. Prencipe, I.; Dellasega, D.; Zani, A.; Rizzo, D.; Passoni, M. Energy dispersive x-ray spectroscopy for nanostructured thin film density evaluation. Sci. Technol. Adv. Mater. 2015, 16, 025007. [CrossRef] [PubMed]

42. Marsh, H.; Rodríguez-Reinoso, F.; Marsh, H.; Rodríguez-Reinoso, F. Characterization of Activated Carbon. Act. Carbon 2006, 143-242. 
43. Shafeeyan, M.S.; Daud, W.M.A.W.; Houshmand, A.; Shamiri, A. A review on surface modification of activated carbon for carbon dioxide adsorption. J. Anal. Appl. Pyrolysis 2010, 89, 143-151. [CrossRef]

44. Lopez-Ramon, M.V.; Stoeckli, F.; Moreno-Castilla, C.; Carrasco-Marin, F. On the characterization of acidic and basic surface sites on carbons by various techniques. Carbon 1999, 37, 1215-1221. [CrossRef]

45. Barczak, M. Amine-modified mesoporous silicas: Morphology-controlled synthesis toward efficient removal of pharmaceuticals. Microporous Mesoporous Mater. 2019, 278, 354-365. [CrossRef]

46. Dobrowolski, R.; Oszust-Cieniuch, M.; Dobrzyńska, J.; Barczak, M. Amino-functionalized SBA-15 mesoporous silicas as sorbents of platinum (IV) ions. Colloids Surf. A Physicochem. Eng. Asp. 2013, 435, 63-70. [CrossRef]

47. Barczak, M.; Dobrzyńska, J.; Oszust, M.; Skwarek, E.; Ostrowski, J.; Zieba, E.; Borowski, P.; Dobrowolski, R. Synthesis and application of thiolated mesoporous silicas for sorption, preconcentration and determination of platinum. Mater. Chem. Phys. 2016, 181, 126-135. [CrossRef]

48. Barczak, M.; Gil, M.; Terpiłowski, K.; Kamiński, D.; Borowski, P. Influence of bridged monomer on porosity and sorption properties of mesoporous silicas functionalized with diethylenetriamine groups. Adsorption 2019, 25, 575-589. [CrossRef]

49. Barczak, M.; Borowski, P. Silica xerogels modified with amine groups: Influence of synthesis parameters on porous structure and sorption properties. Microporous Mesoporous Mater. 2019, 281, 32-43. [CrossRef]

50. Barczak, M.; Dobrowolski, R.; Borowski, P.; Giannakoudakis, D.A. Pyridine-, thiol- and amine-functionalized mesoporous silicas for adsorptive removal of pharmaceuticals. Microporous Mesoporous Mater. 2020, 299, 110132. [CrossRef]

51. Barczak, M. Synthesis and structure of pyridine-functionalized mesoporous SBA-15 organosilicas and their application for sorption of diclofenac. J. Solid State Chem. 2018, 258, 232-242. [CrossRef]

(C) 2020 by the authors. Licensee MDPI, Basel, Switzerland. This article is an open access article distributed under the terms and conditions of the Creative Commons Attribution (CC BY) license (http://creativecommons.org/licenses/by/4.0/). 